\title{
Evidence for a BKT transition and a pseudogap phase in three-dimensional Gross-Neveu model at $T \neq 0$
}

\author{
Costas G. Strouthos ${ }^{\mathrm{a}}$ \\ a Department of Physics, University of Wales Swansea, \\ Singleton Park, Swansea SA2 8PP, United Kingdom
}

\begin{abstract}
We present results from Monte Carlo simulations of the three-dimensional Gross-Neveu Model with a $U(1)$ chiral symmetry at nonzero temperature. We provide evidence that the model undergoes a Berezinskii-KosterlitzThouless transition in accordance with the dimensional reduction scenario. We also identify a regime in the high temperature phase in which the fermions acquire nonzero dynamical mass, analogous to the pseudogap behaviour observed in cuprate superconductors.
\end{abstract}

\section{Introduction}

The three-dimensional Gross-Neveu model $\left(\mathrm{GNM}_{3}\right)$ has been proved to be an interesting and tractable model to study chiral phase transitions both numerically by means of lattice simulations and analytically in the form of large- $N_{f}$ expansions. In this report we present results of numerical simulations of the $U\left(N_{f}\right)_{V}$-invariant $\mathrm{GNM}_{3}$ with a $U(1)$ chiral symmetry at non-zero temperature. This model is described by the following continuum Euclidean Lagrangian density:

$\mathcal{L}=\bar{\Psi}_{i}\left(\not \partial+\sigma+i \gamma_{5} \pi\right) \Psi_{i}+\frac{N_{f}}{2 g^{2}}\left(\sigma^{2}+\pi^{2}\right)$.

We treat $\Psi_{i}, \bar{\Psi}_{i}$ as four-component Dirac spinors and the index $i$ runs over $N_{f}$ fermion species. The model is renormalizable in the $1 / N_{f}$ expansion unlike in the loop expansion [1]. For sufficiently strong coupling at $T=0$ it exhibits spontaneous chiral symmetry breaking and if we choose $\langle\pi\rangle=0$ the pion field $\pi$ is the associated Goldstone boson.

At leading order in $1 / N_{f}$ this model undergoes a second order chiral phase transition [1]. This conclusion is expected to be valid only when $N_{f}$ is strictly infinite, i.e. when the fluctuations of the bosonic fields are neglected, otherwise it runs foul of the Coleman-Mermin-Wagner theorem, which states that in two-dimensional systems the continuous chiral symmetry must be manifest for all
$T>0$. Next-to-leading order calculations [2,3 demonstrated that the the model undergoes a Berezinskii-Kosterlitz-Thouless (BKT) transition [4] in accordance with the dimensional reduction scenario. In [5] it was shown that the thermal transition of the $Z_{2}$-symmetric $\mathrm{GNM}_{3}$ model belongs to the $2-d$ Ising universality class. It is easier to visualize the BKT scenario if we use the "modulus-phase" parametrization $\sigma+i \pi \equiv \rho e^{i \theta}$. In two spatial dimensions logarithmically divergent infrared fluctuations do not allow the phase $\theta$ to take a fixed direction and therefore prevent spontaneous symmetry breaking via $\langle\theta\rangle \neq 0$. The critical temperature $T_{B K T}$ is expected to separate two different chirally symmetric phases: a low $T$ phase, which is characterized by power law phase correlations $\left\langle e^{i \theta(x)} e^{-i \theta(0)}\right\rangle \sim x^{-\eta(T)}$ at distances $x \gg 1 / T$ but no long range order (i.e. a spinwave phase where chiral symmetry is "almost but not quite broken"), and a high $T$ phase which is characterized by exponentially decaying phase correlations with no long range order. In other words for $0 \leq T \leq T_{B K T}$ there is a line of critical points characterised by a continuously varying $0 \leq \eta(T) \leq \frac{1}{4}$. However, since the amplitude $\rho$ is neutral under $U(1)$, the dynamics of the low temperature phase do not preclude the generation of a fermion mass $m_{f} \propto \rho$ whose value may be comparable with the naive prediction of the large- $N_{f}$ approach. In [2] it is shown that in 


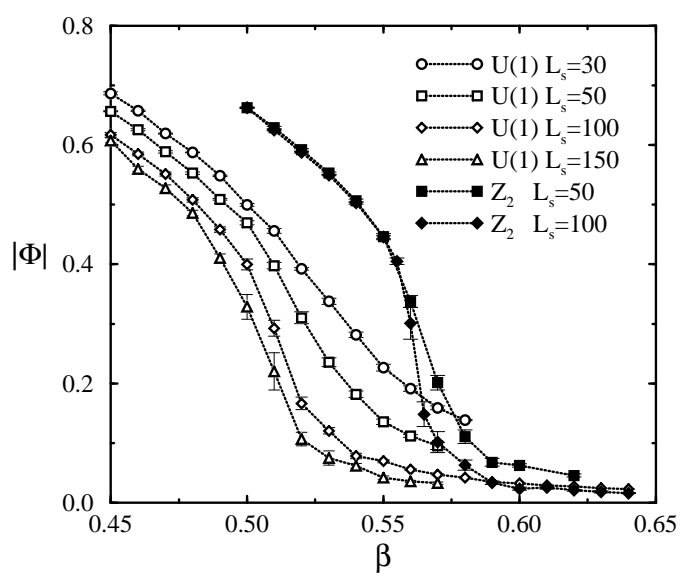

Figure 1. Order parameter $|\Phi|$ vs. $\beta$ for $L_{t}=4$ for both $U(1)$ and $Z_{2}$ symmetric models.

$\mathrm{GNM}_{3}$ with $T>0$, next-to-leading order corrections cause fermion mass generation to occur for $T<T_{*}$, where $T_{*}>T_{B K T}$, i.e. it predicts a "pseudogap" phase in which the system is non-critical. In the next section we present numerical evidence in favour of these scenarios [6].

\section{Results}

The model was formulated on the lattice using the staggered fermion formulation and the simulations were performed using the standard hybrid Monte Carlo algorithm. Details concerning the lattice action and the algorithm can be found in [7].

In order to study the behaviour of the chiral symmetry at $T>0$ in the absence of a fermion bare mass in the Lagrangian the best thing to measure is an effective "order parameter" $|\Phi| \equiv$ $\sqrt{\Sigma^{2}+\Pi^{2}}$, which is a projection onto the direction of $\Phi^{\alpha} \equiv(\Sigma, \Pi)$ separately for each configuration.

In Fig.11 we plot $|\Phi|$ versus $\beta \equiv 1 / g^{2}$ for different lattice sizes together with the results from simulations of the model with a $Z_{2}$ chiral symmetry. At the bulk critical coupling $\beta_{c}^{\text {bulk }} \equiv 1 / g^{2} \approx 0.86$ [6] the lattice spacing becomes zero and $T \rightarrow \infty$. It is clear that the order parameter of the $Z_{2}$ model is independent of the lattice size until just be-

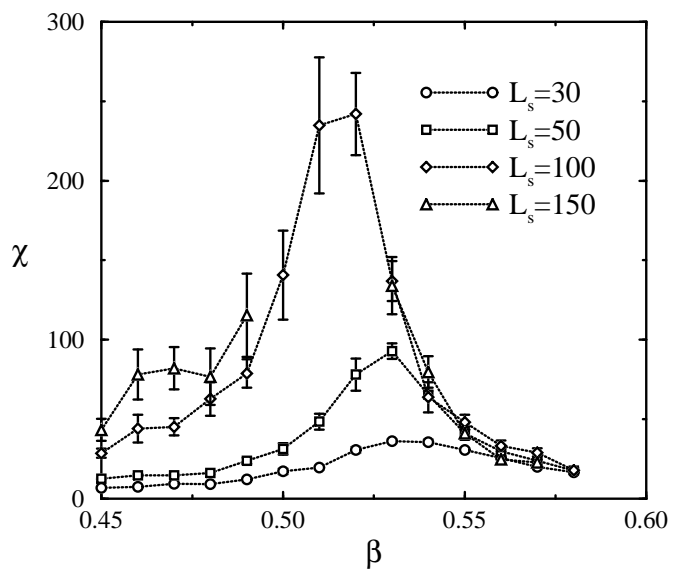

Figure 2. Susceptibility $\chi$ vs. $\beta$.

fore the transition at $\beta=\beta_{c}^{Z_{2}}=0.565(3)$, whereas in the $U(1)$ model $|\Phi|$ has a strong size dependence for a large range of values of $\beta$, i.e. it decreases rapidly as the spatial volume increases in accordance with the expectation that chiral symmetry should be restored for $T>0$. The finite spatial extent $L_{s}$ provides a cut-off for the divergent correlation length and according to the BKT scenario the slow decay of the correlation function $\left\langle e^{i \theta(x)} e^{-i \theta(0)}\right\rangle$ with exponent $\eta(T)<0.25$ for $T<T_{B K T}$ ensures a non-zero magnetization even in a system with very large size. In Fig.2 we plot the susceptibility of the order parameter $\chi=V\left(\left\langle|\Phi|^{2}\right\rangle-\langle|\Phi|\rangle^{2}\right)$, measured on lattices with different spatial size. We observe from this figure that: (a) there is a phase transition since the peak of $\chi$ diverges as we increase $L_{s}$; (b) the transition occurs at a much smaller temperature than the critical temperature of the $Z_{2}$-symmetric model, because the IR fluctuations are stronger in the continuous symmetry case and (c) in the low $T$ phase the susceptibility has a a stronger size dependence than in the high $T$ phase and the errors at low $T$ are much larger than at high $T$. This is strong evidence that the system in critical in the low $T$ phase in accordance with the BKT scenario. We also measured the specific heat $C_{V}$, which we calculated from the fluctuations of the bosonic action $S_{b}=\frac{1}{2} \sum_{x}\left[\sigma^{2}(x)+\pi^{2}(x)\right] . C_{v}$ is 


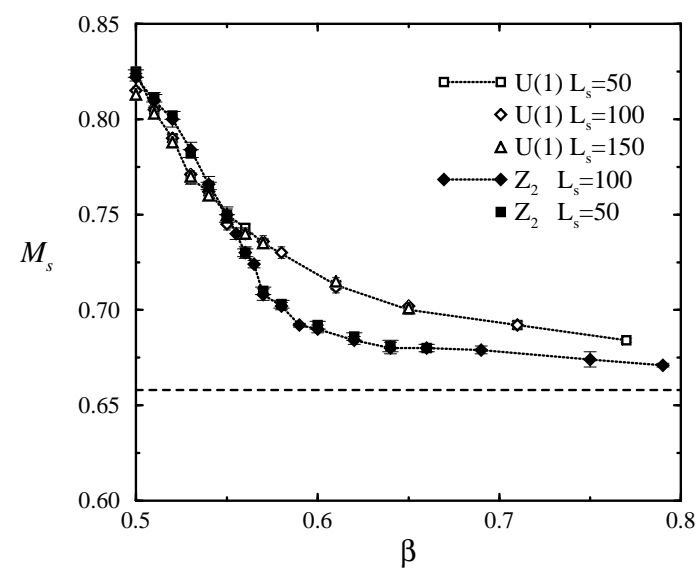

Figure 3. Fermion screening mass $M_{s}$ vs. $\beta$. The horizontal line shows the lattice lowest Matsubara mode $\omega_{0}$.

given by $C_{v}=\frac{\beta^{2}}{V}\left(\left\langle S_{b}^{2}\right\rangle-\left\langle S_{b}\right\rangle^{2}\right)$. We observed that $C_{v}$ has a broad peak at $\beta \simeq 0.50$ and it does not show any divergent behaviour or significant finite size effects. This is also consistent with the BKT scenario, according to which $\alpha=-2$.

Finally, we measured the fermion screening mass $M_{s}(T)$ from the exponential decay of the spatial correlator along one of the spatial lattice axes. The results extracted from the simulations of the $U(1)$ and $Z_{2}$ models on lattices with different $L_{s}$, are shown in Fig.3. If we assume that the temperature effects are absorbed into a temperature dependent pole mass $M(T)$ and a "renormalization" of the speed of light coefficient $A(T)$ then we have $M_{s}^{2}(T)=A(T)\left[M^{2}(T)+\omega_{0}^{2}\right]$. We can infer from Fig. 3 that the screening mass is independent of $L_{s}$ for almost all values of $\beta$. In the $Z_{2}$ case we ascribe mass generation to orthodox chiral symmetry breaking. The similarity of $M_{s}^{U(1)}$ and $M_{s}^{Z_{2}}$ at low $T$ where $A \approx 1$ suggests that the pole mass $M(T)$ is nonzero. By comparing Fig.2 with Fig. 3 we infer that the fermions are massive in the phase where both chiral symmetry is restored and the system is not critical. This implies the existence of a pseudogap phase for $\beta_{B K T} \leq \beta \leq \beta_{c}^{Z_{2}}$. The deviation of $M_{s}(T)$ from $\omega_{0}$ for $\beta>0.56$ could be attributted to either $A(T)>1, M(T)>0$ or discretization effects.
To understand the physics in this regime these effects need to be disentangled via a detailed study of the fermion dispersion relation closer to the continuum limit on lattices with $L_{t} \geq 8$. For this reason we cannot at this stage identify a second phase transition where mass generation switches off.

\section{Summary and Outlook}

The numerical results presented in this report provide strong evidence that the nonzero temperature phase structure of the $U(1)$-symmetric $\mathrm{GNM}_{3}$ is consistent with the BKT scenario. We have also shown that there is a pseudogap phase i.e., the fermion mass remains nonzero above $T_{B K T}$. We are currently extending our work in various directions. We wish (i) to increase our statistics in order to extract the exponents $\eta(T)$; (ii) to study the fermion dispersion relation and (iii) to understand the $N_{f}$ dependence of our various results.

\section{Acknowledgements}

This project is being done in collaboration with Simon Hands and John Kogut. Costas Strouthos is supported by a Leverhulme Trust grant.

\section{REFERENCES}

1. B. Rosenstein, B.J. Warr and S.H. Park, Phys. Rep. 205 (1991) 59.

2. E. Babaev, Phys. Lett. B497 (2001) 323; E. Babaev, Int. J. Mod. Phys. A16 (2001) 1175.

3. H. Yamamoto and I. Ichinose, Nucl. Phys. B370, (1992) 695; T. Appelquist and M. Schwetz, Phys. Lett. B491 (2000) 367.

4. V.L. Berezinskii, Zh. Eksp. Teor. Fiz. 59 (1970) 907 [Sov. Phys., JETP 32 (1971) 493]; J.M. Kosterlitz and D.J. Thouless, J. Phys. C6 (1973) 1181.

5. J.B. Kogut, M.A. Stephanov and C.G. Strouthos, Phys. Rev. D58 (1998) 096001.

6. S.J. Hands, J.B. Kogut and C.G. Strouthos, Phys. Lett. B515, (2001) 407.

7. S.J. Hands, A. Kocic and J.B. Kogut, Ann. Phys. 224 (1993) 29; S.J. Hands, S. Kim and J.B. Kogut, Nucl. Phys. B442 (1995) 335. 